\title{
Intravenous aspirin challenge as a diagnosis of nonsteroidal anti-inflammatory drugs hypersensitivity
}

\author{
Gil Myeong Seong, Jaechun Lee, Changhwan Kim
}

\begin{abstract}
Background: Nonsteroidal anti-inflammatory drugs (NSAIDs) are a major cause of drug-induced hypersensitivity, called "NSAID hypersensitivity". A confirmative diagnosis is necessary for ensuring drug safety and finding alternative drugs. No reliable test other than direct challenge is diagnostic. An intravenous (IV) aspirin challenge has rarely been tried.

Objective: To assess the safety and efficacy of the aspirin IV provocation test.

Methods: A retrospective and descriptive study in a hospital. Clinical data were reviewed in patients who had aspirin IV provocation test with lysine aspirin.

Results: In 71 patients suspected of having NSAID hypersensitivity, aspirin IV provocation test was performed. Most provocations were performed on the same day. Forty-three $(60.6 \%)$ showed a positive response to the challenge. The positive reactions were rescued mostly by antihistamines or glucocorticoids and rarely with bronchodilators and epinephrine. Three patients who showed a negative response to the aspirin challenge were shown to have single-NSAID hypersensitivity. For confirmation of NSAID hypersensitivity in these patients, the sensitivity of the IV aspirin provocation test was $93.5 \%$.
\end{abstract}

Conclusions: Aspirin IV provocation test with lysine aspirin on the same day is safe and efficacious for diagnosing NSAID hypersensitivity.

Key words: Anti-Inflammatory Agents, Non-Steroidal; Aspirin; Diagnosis; Hypersensitivity; Drug hypersensitivity

\section{From:}

Department of Internal Medicine, Jeju National University School of Medicine, Jeju, Korea

\section{Introduction}

Analgesics are the most widely consumed medicines. Nonsteroidal anti-inflammatory drugs (NSAIDs) are most widely used analgesics as well as one of the most common causes of drug-induced hypersensitivity, called "NSAID hypersensitivity". ${ }^{1}$ Diverse and variable manifestations that range from an itchy nose to life-threatening anaphylaxis have been reported. For safety reasons, a confirmative diagnosis should be established to provide patients with a list of drugs to be avoided and to suggest alternatives. ${ }^{2}$

A reliable in vitro test or predictive marker is still under investigation for the common clinical condition of NSAID hypersensitivity. ${ }^{3}$ Direct provocation with aspirin or with the NSAIDs of interest is the only decisive diagnostic test. Oral, bronchial, or nasal routes of aspirin administration for the provocation have been suggested,,$^{1,4,5}$ but intravenous (IV) provocation is rarely attempted.,
Corresponding author:

Jaechun Lee

Department of Internal Medicine, Jeju National University School of Medicine, 102 Jejudaehak-ro, Jeju 63243 South Korea

E-mail: doc4u@hanmail.net

In the management of patients with NSAID hypersensitivity, proper choice of safe analgesics is crucial. Acetaminophen or a cyclooxygenase-2 (COX-2) selective inhibitor is suggested, however, the alternative may not always be guaranteed to be safe because of the risk of cross-reactivity. ${ }^{8}$

We had been performed aspirin IV provocation tests with lysine aspirin, retrospectively assessed to determine its safety and efficacy for the diagnosis of NSAID hypersensitivity.

\section{Methods}

We retrospectively reviewed the clinical data of subjects who had taken the aspirin provocation test using lysine aspirin from January 2012 to December 2017 in Jeju National University Hospital, Jeju, Korea. (IRB \#2013-12-007) 


\section{Subjective}

Patients suspected of having NSAID hypersensitivity were asked to undergo the provocation. The patients were not allowed to take any medicine including bronchodilators, glucocorticoids, antihistamines or leukotriene receptor antagonists for at least 1 week before the test.

\section{Provocation protocol (Figure 1)}

The aims of the aspirin IV provocation test were to make a confirmative diagnosis of NSAID hypersensitivity. At least 1 month after a previous hypersensitivity episode, the patient was admitted to a day surgery center (DSC) or general ward (GW) upon a physician's decision and with the patient's informed consent. In the DSC, the patient is meant to be discharged on the same day of admission, whereas in the GW, the patient may stay for at least 1 night. At admission, vital signs were monitored, and IV saline was connected through a peripheral venous catheter. Patients were told to report any symptoms or signs occurring during the provocation test and were closely observed by a nurse.

71 Patients having suspicious NSAIDs-related symptoms/signs

\section{- > 1 month after completion of symptoms/signs \\ - Informed consent}

Aspirin intravenous provocation test with serial lysine aspirin

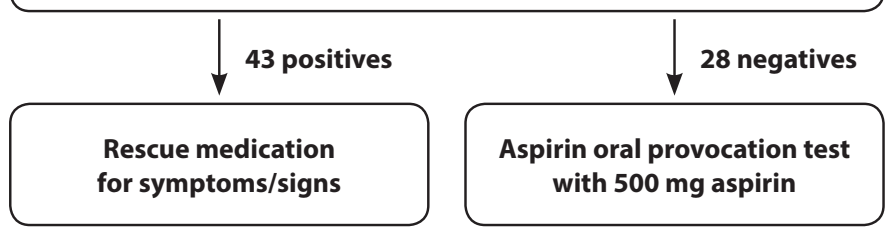

Figure 1. The scheme of the study flow.

During the test, any occurrence of symptoms or signs such as itching, rhinorrhea, shortness of breath, wheezing, abdominal pain, chest discomfort, dizziness, fainting, hives, angioedema, hypotension, or anaphylaxis was considered as a positive reaction. If a patient displayed any symptom or sign, the test was halted, and the patient was managed according to the symptoms or signs.

Lysine-acetylsalicylic acid (L-aspirin, Arthalgyl injection, Il-Yang Pharm, Gyeonggi, Korea) was given IV as a bolus at 30 min intervals; the equivalent amounts of aspirin were $9 \mathrm{mg}$, $22.5 \mathrm{mg}, 45 \mathrm{mg}, 90 \mathrm{mg}, 225 \mathrm{mg}$, and $450 \mathrm{mg}$. The cumulative dose of aspirin was $841.5 \mathrm{mg}$. As an oral aspirin provocation, aspirin $500 \mathrm{mg}$ was given orally, and the patient was observed for $2 \mathrm{~h}$. For patients who responded negatively, the total time of the entire test was about $7 \mathrm{~h}$ (Table $\mathbf{1}$ ).
Table 1. The actual protocol of the provocation test.

\begin{tabular}{|ccc|}
\hline \multicolumn{2}{|c}{ Lysine-aspirin } & \multirow{2}{*}{ Aspirin } \\
\cline { 1 - 2 } Bolus IV & Cumulative dose & \\
\hline $9 \mathrm{mg}$ & $9 \mathrm{mg}$ & $500 \mathrm{mg}$ orally \\
$22.5 \mathrm{mg}$ & $31.5 \mathrm{mg}$ & $* 2 \mathrm{~h}$ interval \\
\hline $45 \mathrm{mg}$ & $76.5 \mathrm{mg}$ & \\
\hline $90 \mathrm{mg}$ & $166.5 \mathrm{mg}$ & \\
\hline $225 \mathrm{mg}$ & $391.5 \mathrm{mg}$ & \\
\hline $450 \mathrm{mg}$ & $841.5 \mathrm{mg}$ \\
& $* 30 \mathrm{~min}$ interval \\
\hline
\end{tabular}

\section{Definition of anaphylaxis}

Anaphylaxis is defined as an acute onset of symptoms involving two or more systems within minutes or hours as described in the literature. ${ }^{9}$ The involvement is of the skin or mucosal surface, respiratory compromise, hypotension, or persistent gastrointestinal symptoms. Accordingly, generalized hives and angioedema (skin/mucosal involvement only) was not considered to be anaphylactic, whereas angioedema with shortness of breath and chest discomfort (skin/mucosal and respiratory involvement) was considered to be anaphylactic.

\section{Results}

The provocation test was performed in 71 patients $(67.6 \%$ women) whose mean age was $42 \pm 17$ years (range 15-83). Forty-eight $(67.6 \%)$ underwent the provocation test in the DSC. The records of previous adverse reactions of these patients showed that 34 patients $(47.9 \%)$ had experienced anaphylaxis. The skin/mucosal system (70.4\%) was commonly involved, followed by the respiratory $(32.4 \%)$, cardiovascular $(32.4 \%)$, nervous (4.2\%), and gastrointestinal (2.8\%) systems. Prescriptions to counter the adverse reactions were provided and reviewed completely for 53 patients $(74.6 \%)$. Only 12 patients (16.9\%) had experienced a reaction to a single drug (NSAID),

Table 2. Demographics of 71 enrolled subjects.

\begin{tabular}{lc}
\hline Age, year-old & $42 \pm 17(15-83)$ \\
Sex, women & $48(67.6 \%)$ \\
History of adverse reaction & \\
Anaphylaxis & $34(47.9 \%)$ \\
Skin/mucosal system & $50(70.4 \%)$ \\
Gastrointestinal system & $2(2.8 \%)$ \\
Cardiovascular system & $23(32.4 \%)$ \\
Respiratory system & $23(32.4 \%)$ \\
Nervous system & $3(4.2 \%)$ \\
Prescription with adverse reaction provided & $53(74.6 \%)$ \\
Medication with a single component & $12(16.9 \%)$ \\
\hline
\end{tabular}

Data are presented as mean \pm SD (range) or no. of patients (\%). 
and the others had reacted to multiple drugs or could not provide information about their prescriptions (Table 2).

Among the 71 patients who had the aspirin IV provocation test, 43 patients $(60.6 \%, 65.1 \%$ women, mean age $36 \pm 14$ years) tested positive. In the positives, the reaction occurred at a mean cumulative dose of aspirin of $159.2 \mathrm{mg}( \pm 200.0$ $\mathrm{mg}$, range $31.5-841.5 \mathrm{mg}$ ) (Figure 2). Twenty-nine were tested in the DSC, and 28 were discharged on the same day. A 40-year-old woman exhibited shortness of breath at a cumulative dose of aspirin of $391.5 \mathrm{mg}$. Her symptom was not completely reversed within hours, and she was moved to the GW to stay for another day. The following drugs were used to reverse the reaction: IV antihistamines in 38 patients (88.4\%), IV glucocorticoids in 27 (62.8\%), salbutamol inhalation in 4 (9.3\%), intramuscular epinephrine in $2(4.7 \%)$, IV metoclopramide in $1(2.3 \%)$, and oxygen supplementation in $1(2.3 \%)$. Two patients (4.7\%) needed no medication.

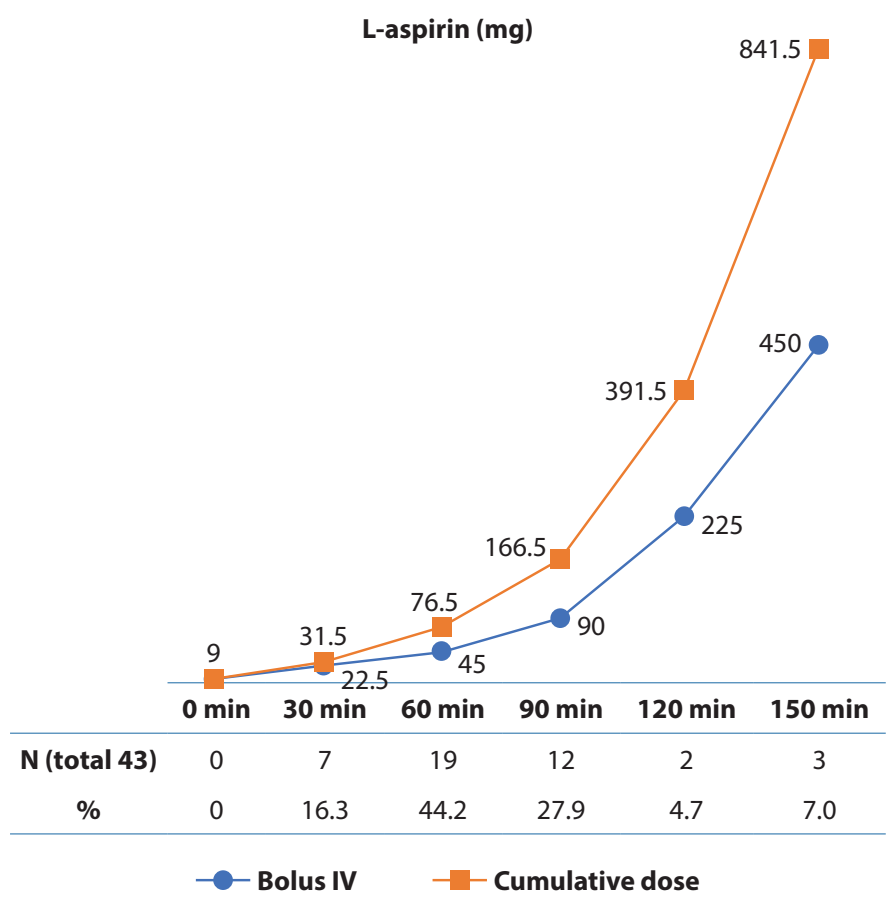

Figure 2. The provocative and cumulative doses of lysine aspirin in 43 patients with positive intravenous aspirin provocation test.

Among the 28 patients who were negative in the provocation $(71.4 \%$ women, mean age $51 \pm 17$ years), no one reacted to the consecutive oral challenge with $500 \mathrm{mg}$ of aspirin. The other culprits were successfully proven later in 10 patients. (3 patients with NSAID hypersensitivity, 2 with acetaminophen hypersensitivity, 2 with beta-lactam drug hypersensitivity, 1 with chlorphenesin carbamate hypersensitivity, and 2 idiopathic angioedema patients). Two patients were diagnosed as acetaminophen positive and IV aspirin negative, and three patients were diagnosed with "single" NSAID hypersensitivity.

A 58-year-old man had previously experienced anaphylaxis only with zaltoprofen. An oral provocation test with zaltoprofen was not performed for safety reasons. A 55-year-old woman had previously experienced repeated anaphylaxis with medicines including diclofenac or aceclofenac. Anaphylaxis was reproduced during an intradermal test with diclofenac. A 36-year-old woman had previously experienced anaphylaxis only with ibuprofen. She reacted positively to a skin prick test with ibuprofen. The last two patients might be considered to have NSAID hypersensitivity with an immunological mechanism.

For diagnosing NSAID hypersensitivity, the sensitivity of the aspirin provocation test with lysine aspirin might be $93.5 \%$, and the specificity $100 \%$; this included no false positives and 3 false negative cases with single-NSAID hypersensitivity, who did not react to the consecutive aspirin oral challenge (Table 3).

Table 3. Safety and efficacy of aspirin intravenous provocation test

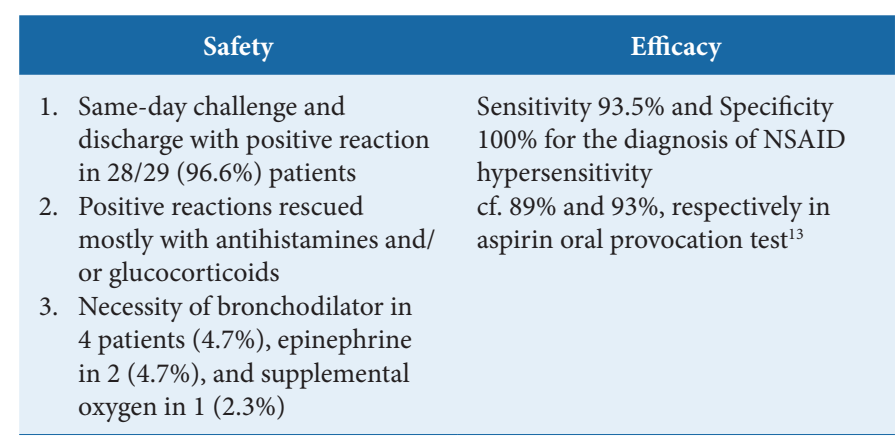

NSAID, Nonsteroidal anti-inflammatory drug.

\section{Discussion}

Adverse drug reactions to NSAIDs are the most prevalent type of drug reaction, mainly because of the wide consumption of these drugs. The mechanism responsible for NSAID hypersensitivity was not clearly understood, but the wellknown cross-reactivity between various NSAIDs is consistent with the shared inhibition of cyclooxygenase-1. In a scanty portion of people with NSAID hypersensitivity, the mechanism is immunologically related, as shown by the lack of cross -reactivity with different NSAIDs or positive skin testing with NSAIDs. ${ }^{1}$ A wide range of genetic and epigenetic explanations have been proposed, but these are seldom applied to the diagnosis and prediction of his common clinical condition. ${ }^{3}$

The oral provocation test with aspirin was first introduced in the mid-1970s, ${ }^{10}$ and is now considered to be the gold standard for a confirmative diagnosis. ${ }^{1}$ Despite the lack of a standard protocol, the suggested cumulative aspirin dose is accepted as $1000 \mathrm{mg}$, starting at a dose of $1 / 10$ or less and increasing at intervals of 1.5 to $2 \mathrm{~h} .{ }^{11}$ The total time for the test depends on the intervals used in the challenge. Because of the risk of a severe reaction, the total time of some protocols can be 1 or more days. ${ }^{4}$

As a multi-systemic challenge, an oral aspirin challenge is the most sensitive method for a confirmative diagnosis. Diverse multi-systemic symptoms and signs are expected. By contrast, local aspirin provocation may be confined in local symptoms and signs as a positive reaction. Therefore, respiratory challenge through the nasal/bronchial route is not recommended in the diagnosis of NSAID-exacerbated urticarial/angioedema. ${ }^{1}$ 
Despite the lower sensitivity than in oral provocation, nasal/ bronchial aspirin provocation with lysine aspirin may be chosen for its relative safety. ${ }^{4}$

The IV route is the easiest means of prompt drug delivery at a given time. For the prejudice of occurring severe adverse reaction, the IV aspirin provocation test has not been used often in clinical practice. A multi-systemic challenge as well as prompt reaction are expected to have a high sensitivity with a shorter duration of provocation. The safety and efficacy of this convenient method has not been assessed.

Mita et al. $^{6}$ attempted IV provocation with lysine aspirin in 7 patients with allegedly aspirin-intolerant asthma. At a cumulative IV aspirin dose of as little as $25-75 \mathrm{mg}$, all exhibited a $20 \%$ decrease in lung function. The authors applied this protocol in another 12 patients with aspirin-intolerant asthma. ${ }^{12}$ In a patient with NSAID-exacerbated chronic urticaria, the IV challenge with lysine aspirin provoked extensive hives during the desensitization process. ${ }^{7}$

In this study, we used the IV provocation test with lysine aspirin in 71 patients suspected of having NSAID hypersensitivity. In the 43 positive patients, the mean provocative dose of aspirin was $<200 \mathrm{mg}$, although there was a wide range. The decision of the positivity mainly depended on the patients' report with any occurrence of symptoms and signs. If a patient exhibited obscure or ambiguous symptoms or signs, the protocol proceeded cautiously to the next step to have objective validity. No patient died, and some severe reactions were medically reversed with ease. Up to half of the patients included in this study had previously experienced anaphylaxis, as noted in the medical record. Kim et al. ${ }^{8}$ administered an oral aspirin provocation test with a cumulative dose of aspirin up to $900 \mathrm{mg}$ in 180 patients, including 31 with a history of anaphylaxis. The challenge-related anaphylaxis was not described for the 131 positive patients. The safety of the test was objectively ensured by the use of reverse or rescue medications. In our investigation, antihistamines and systemic glucocorticoids were frequently necessary for patients with a positive reaction, but epinephrine was needed by only 2 patients. Although a history of anaphylaxis is a contraindication for an oral aspirin challenge, ${ }^{3}$ we cautiously propose that an IV aspirin challenge might be applicable.

IV aspirin provocation might be more efficacious over aspirin oral challenge in the diagnosis of NSAID hypersensitivity. Mita et al.,12 reproduced bronchospasm in all aspirinintolerant asthmatics, and the sensitivity reached $100 \%$. Nizankowska et al. ${ }^{13}$ reported that the sensitivity and the specificity of oral challenge with 500mg of cumulative aspirin were $89 \%$ and $93 \%$, respectively, with $20 \%$ fall of FEV1 and extra-bronchial symptoms together. No patient reacted to 500 $\mathrm{mg}$ in the oral aspirin challenge in this study. Once classified with aspirin exacerbated respiratory disease, some patient may declare itching in the mouth, runny nose, or swollen eyelids prior to the respiratory distress when exposed to NSAIDs. In spite of including heterogeneous phenotypes in the reactions and considering diverse reactions as a positive, for excluding NSAID hypersensitivity, our protocol reached the highest specificity, except for rare immune-related cases of singleNSAID hypersensitivity.
Hypersensitivity to a single NSAID is an extremely rare condition that has been described only in case reports. Different from the more common condition of cross-reactivity, the mechanism for single-NSAID hypersensitivity may involve an IgE-mediated immunological reaction. ${ }^{1}$ In patients with this rare type of hypersensitivity, a skin prick or intradermal test with the affected NSAID before the IV aspirin provocation test would be diagnostic.

Once NSAID hypersensitivity is strongly suspected or documented by provocation, the next challenge is to select alternative analgesics for the patient. Acetaminophen and/or COX-2-selective inhibitors are commonly recommended. In a large-scale study of oral challenge-proven aspirin-hypersensitive patients in Korea, acetaminophen and celecoxib were tested orally and caused positive tests in $25 \%$ and $10 \%$ of patients, respectively. ${ }^{8}$

In conclusion, The IV aspirin provocation test with lysine aspirin appears to be safe and efficacious for diagnosing NSAID hypersensitivity. A larger-scale trial should be undertaken to confirm these results.

\section{Acknowledgments}

none

\section{Conflict of interest}

No potential conflict of interest relevant to this article was reported.

\section{References}

1. Kowalski ML, Makowska J, Blanca M, Bavbek S, Bochenek G, Bousquet J, et al. Hypersensitivity to nonsteroidal anti-inflammatory drugs (NSAIDs) -classification, diagnosis and management: review of the EAACI/ENDA and GA2LEN/HANNA. Allergy. 2011;66:818-29.

2. Aberer W, Bircher A, Romano A, Blanca M, Campi P, Fernandez J, et al. Drug provocation testing in the diagnosis of drug hypersensitivity reactions: general considerations. Allergy. 2003;58:854-63.

3. Le Pham D, Kim J-H, Trinh THK, Park H-S. What we know about nonsteroidal anti-inflammatory drug hypersensitivity. Korean J Intern Med. 2016;31:417-32.

4. Niżankowska E, Bochenek G, Mastalerz L, Świerczyńska M, Picado C, Scadding G, et al. EAACI/GA2LEN guideline: aspirin provocation tests for diagnosis of aspirin hypersensitivity. Allergy. 2007;62:1111-8.

5. Stevenson DD, Szczeklik A. Clinical and pathologic perspectives on aspirin sensitivity and asthma. J Allergy Clin Immunol. 2006;118:773-86.

6. Mita H, Higashi N, Taniguchi M, Higashi A, Akiyama K. Increase in urinary leukotriene B4 glucuronide concentration in patients with aspirin-intolerant asthma after intravenous aspirin challenge. Clin Exp Allergy. 2004;34:1262-9.

7. Lee J. Aspirin desensitization as a treatment for aspirin-sensitive chronic spontaneous urticaria. Dermatol Ther. 2015;28:4-6.

8. Kim Y-J, Lim K-H, Kim M-Y, Jo E-J, Lee S-Y, Lee S-E, et al. Cross-reactivity to acetaminophen and celecoxib according to the type of nonsteroidal anti-inflammatory drug hypersensitivity. Allergy Asthma Immunol Res. 2014;6:156-62.

9. Kemp SF, Lockey RF, Simons FE, World Allergy Organization ad hoc committee on epinephrine in anaphylaxis. Epinephrine: The drug of choice for anaphylaxis. A statement of the World Allergy Organization. World Allergy Organ J. 2008;1(suppl 2):S18-26.

10. Szczeklik A, Gryglewski R, Czerniawska-Mysik G. Clinical patterns of hypersensitivity to nonsteroidal anti-inflammatory drugs and their pathogenesis. J Allergy Clin Immunol. 1977;60:276-84.

11. Kowalski M, Asero R, Bavbek S, Blanca M, Blanca-Lopez N, Bochenek $\mathrm{G}$, et al. Classification and practical approach to the diagnosis and management of hypersensitivity to nonsteroidal anti-inflammatory drugs. Allergy. 2013;68:1219-32. 
12. Mita H, Higashi N, Taniguchi M, Higashi A, Kawagishi Y, Akiyama K. Urinary 3-bromotyrosine and 3-chlorotyrosine concentrations in asthmatic patients: lack of increase in 3-bromotyrosine concentration in urine and plasma proteins in aspirin-induced asthma after intravenous aspirin challenge. Clin Exp Allergy. 2004;34:931-8.
13. Nizankowska E, Bestynska-Krypel A, Cmiel A, Szczeklik A. Oral and bronchial provocation tests with aspirin for diagnosis of aspirin-induced asthma. Eur Respir J. 2000;15:863-9. 